\title{
ANOMALOUS HORIZONTAL LAMINATION OF NERVE CELLS IN THE SUPRAGRANULAR CORTEX OF AN IDIOT BRAIN
}

\author{
BY \\ M. C. H. DODGSON \\ From the Department of Neuropathology, Institute of Psychiatry, Maudsley Hospital, London, and the \\ Fountain Hospital, London
}

Except in the gross developmental anomalies, such as, for instance, microgyria, unequivocal signs of morphological immaturity in the cerebral cortex are not easily demonstrated.

\section{Case Reports}

Idiot Cortex.-The following case illustrates the findings in an idiot brain.

Case 1.-David M. was the only child of healthy parents of above the average intelligence, who were first cousins. There was no history of mental deficiency in the family. Delivery was by forceps three weeks before term, following an apparently normal pregnancy, and no obstetrical difficulties were encountered. An anaesthetic, thought to be chloroform, was given to the mother during the delivery. The child was slightly jaundiced during the first week of life, but there were no symptoms of cerebral irritation. His early progress was not satisfactory, and by the tenth day it was thought that he would be mentally deficient. At the age of 18 weeks he was admitted to King's College Hospital for investigation under Dr. Sheldon. Here he was found to have a small head, diameter $16 \frac{1}{2}$ in., and an "ugly M.D. look". Abnormalities were found on examination of the central nervous system. The pupils did not react to light, nor did the eyes follow a light. No attempt was made to grasp an object. There was nystagmus. The optic discs were rather pale. It was thought that hearing was present. The arms were very spastic, with large musculature, the tendon reflexes being very brisk and bilaterally equal. The legs were also very spastic, especially the right. The knee and ankle tendon reflexes were brisk and bilaterally equal, the plantar reflexes extensor.

The following tests were done at King's College Hospital : creatine excretion, $38 \mathrm{mg}$. in 24 hours; plasma cholesterol, $148 \mathrm{mg} . \%$; creatine excretion, $47 \mathrm{mg}$. in 24 hours.

He was admitted to the Fountain Hospital as a mental defective at the age of $2 \frac{1}{4}$ years, and died on April 25, 1949 , within a fortnight of admission.

At necropsy the body was found to be that of a wellnourished male child. The head circumference was $17 \frac{1}{2}$ in.; the skull was long and narrow, with closed fontanelles. The meninges and cerebral venous sinuses appeared normal. The air passages were clear and the pleurae dry. Both lobes of the left lung were congested and heavy, with scattered patches of confluent bronchopneumonia. The right lung was congested. The gastrointestinal tract appeared normal. No abnormality was found in the endocrine system. There was a bilateral downward dislocation of the heads of the femora ; the acetabula was imperfectly formed.

The unfixed brain weighed $282 \mathrm{~g}$. The cerebral hemispheres were narrow in relation to their frontooccipital length, and were perceptibly firmer in consistency than normal. There was no microgyria. The corpus callosum was represented by a thin white membrane extending backwards for a distance of about $30 \mathrm{~mm}$. from the site of the genu.

A detailed examination of the brain was made after it had been fixed in $10 \%$ formol saline. It was bilaterally symmetrical, and apart from the obvious narrowness of the cerebral hemispheres, of grossly normal proportions. The olfactory and other cranial nerves appeared normal. The dimensions of the cerebrum were : frontooccipital length $150 \mathrm{~mm}$.; greatest breadth (mid-parietal) $100 \mathrm{~mm}$. ; greatest height $80 \mathrm{~mm}$. The midbrain, pons, cerebellum, medulla, spinal cord, meninges, and cerebral vessels were of normal appearance.

From coronal sections made through the cerebrum at the level of the tip of the caudate nuclei (Fig. 1),

\footnotetext{
Fig. 1.-Coronal section of brain at level tip of
} caudate nuclei.

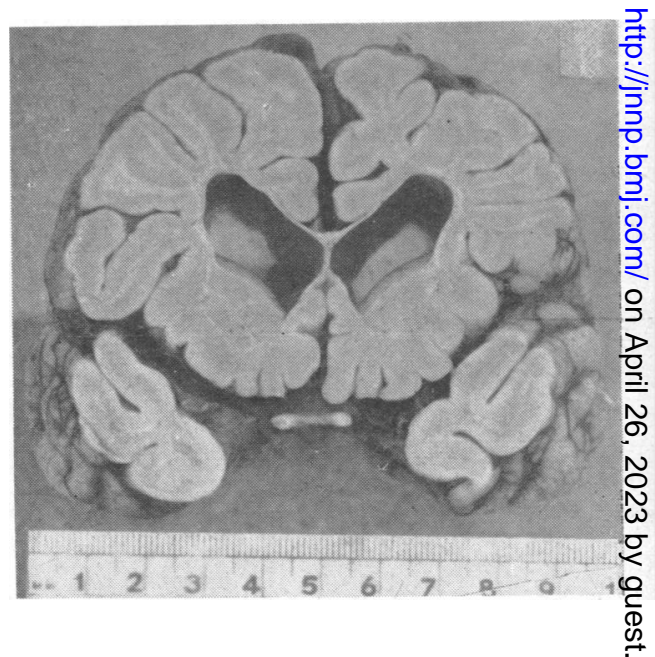


through the parietal region just posterior to the basal ganglia and through the occipital lobe, it could be seen that there was a generalized and symmetrical dilation of the lateral ventricles. The choroid plexuses appeared normal. Associated with the internal hydrocephalus there was a gross deficiency in the amount of central white matter, of about the same degree throughout. The extreme thinness of the corpus callosum was also apparent. In the depths of the sulci the cortical grey matter was separated from the lateral ventricle by a distance of about $4 \mathrm{~mm}$., each gyrus possessing a central core of white matter of about the same width. In the central core of many of the gyri, and particularly at the confluence of two or more, the white matter contained small, pinkish-grey zones in which minute blood vessels could be seen with the naked eye.

The central block, containing the basal ganglia, was divided sagittally. The appearances on either side were identical. The interventricular foramina were abnormally wide, being $7 \mathrm{~mm}$. in diameter. The third ventricle was moderately dilated, the lamina terminalis being of paper thinness. On the other hand, the aqueduct of the midbrain did not appear to be dilated, with a diameter of $1 \mathrm{~mm}$. The fourth ventricle was of normal appearance, and the lateral recesses were patent. The thalamus was small, with an antero-posterior length of $18 \mathrm{~mm}$. There was no anterior tubercle, this accounting in part for the abnormal width of the interventricular foramen. There was no massa intermedia. The relatively small size of the thalamus resulted in some distortion and foreshortening of the hind part of the third ventricle, the hypothalamic sulcus running at an angle of $45^{\circ}$ to the horizontal. The septum lucidum was extremely thin, and the fornix system could not be identified, while the mammillary body was small and inconspicuous. On the other hand, the projection of the caudate nucleus into the lateral ventricle appeared to be of normal size in relation to the size of the brain.

Frozen sections were cut from blocks from the superior, orbital, and lateral parts of the left frontal lobe. Blocks for celloidin embedding were also taken from the frontal, temporal, parietal, central, and occipital parts of the right cerebral hemisphere, and from the right basal ganglia, brain stem, pons, medulla, cerebellum, and spinal cord. The frozen sections were stained by all the usual histological methods, the celloidin sections by Nissl's, Heidenhain's myelin, and Holzer's glial fibre methods.

The most unusual feature of this brain was the presence of a cytoarchitectural anomaly in the outer layers of the cerebral cortex, which was most conspicuous in the superior frontal region. In place of their normal, poorly defined radial arrangement, the cells of the second and third cortical layers were distributed in rows of single cells running parallel with the surface of the brain (Figs. 2 and 3). The nerve cells themselves showed signs of immaturity, having rather scanty cytoplasm which contained very little Nissl substance, and were in many instances bipolar, in this respect morphologically resembling neuroblasts. With the exception of radial fibres in the deeper layers, there were very few myelinated fibres in the cortex. A similar
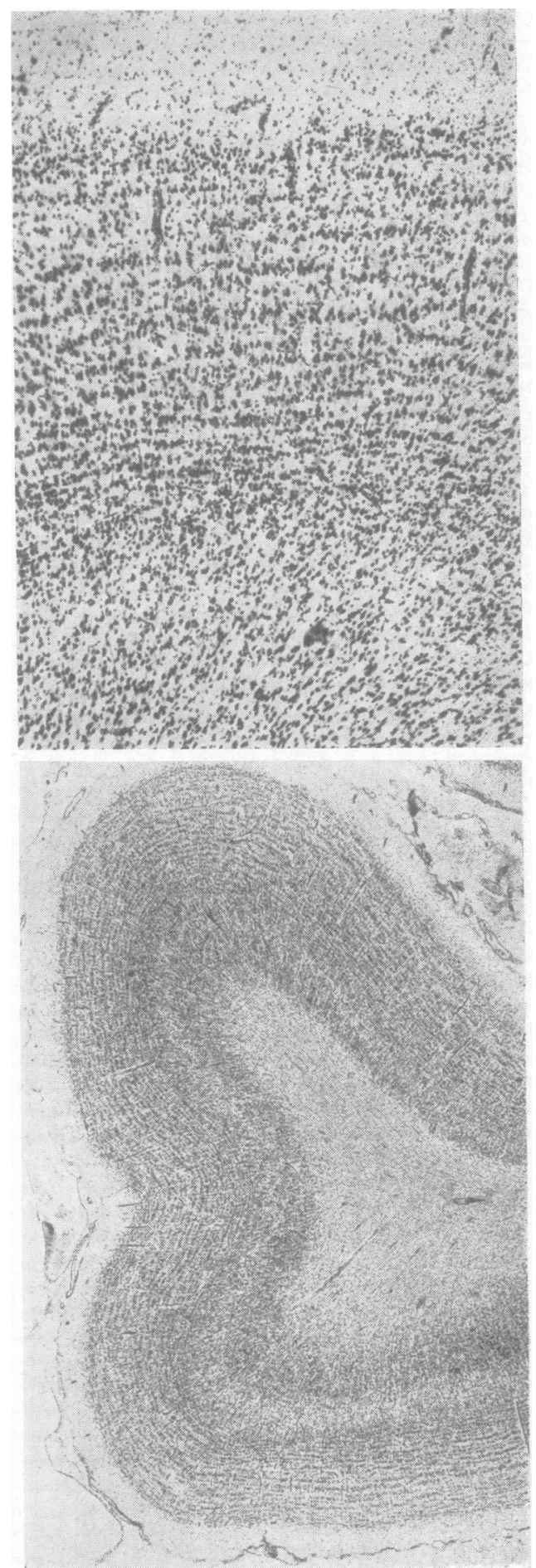

Fig. 2.-Cortex of superior frontal gyrus. Nissl. $\times 50$. Fig. 3.-Same as Fig. 2. $\times 14.5$. 
sparseness of neurofibrils was seen in silver preparations. No fibres appeared to run horizontally between the rows of cells in the outer layers. Although most conspicuous in the prefrontal cortex, the anomalous arrangement of nerve cells was not entirely confined to this part. Except in the central region, a tendency to horizontal lamination, particularly in the outer margin of the second layer, could be detected in all regions of the neocortex. In addition, the first cortical layer was unusually wide throughout, containing large Cajal nerve cells which lay, in some instances, in abnormal positions. A peculiar arrangement of cells was found also in the palaeocortex, where the dentate gyrus was reduplicated, consisting of two closely apposed parallel rows of cells, the outer row being less regular than the inner. In the pyramidal cell layer of the cornu Ammonis the normal crowding of cells in its inner part was absent. No abnormality was noted in the entorhinal cortex.

Immediately beneath the ependyma of both hemispheres there was a narrow zone of dense glial fibrosis, containing relatively few astrocytic nuclei. Subependymal gliosis was also found in the wall of the third ventricle. Contrasting with this there was a densely cellular zone occupying the greater part of the thickness of the shrunken central white matter, containing an excess of astrocytes and oligodendroglial cells and occasional microglial cells, which was the site of a fine glial fibrosis. This zone also contained a large number of neuroblasts scattered throughout the affected white matter. The blood vessels in the central zone, although not structurally altered, were more numerous than in the normal and appeared to be widely dilated. There was no perivascular inflammatory reaction.

There were very few poorly myelinated fibres immediately beneath the ependyma, in the zone corresponding to that in which there was the most dense glial fibrosis. Elsewhere throughout the central white matter, except in the U-fibre systems, myelination was scanty, the myelinated fibres tending to run in poorly defined stripes parallel to the ventricle (Fig. 4). The fibres in the deep white matter of the frontal lobe tended to run together in a diffuse bundle, or in a series of concentric lamellae, apparently contributing fibres to the majority of the superjacent gyri. Posteriorly this system of fibres appeared to be continuous with the external capsule. In the central region the central cores of the gyri were more completely myelinated than elsewhere. The individual myelin sheaths were not apparently abnormal, and there was no indication of recent myelin breakdown. The histological findings seemed to suggest that incomplete myelination had occurred, rather than that there had been a true demyelination.

Significant findings elsewhere in the brain may be mentioned briefly. Irregular myelination had occurred in the thalamus, particularly in the dorsomedial nucleus, with the production of an abortive status marmoratus. In the myelin picture, the anterior, dorsomedial and lateral nuclear groups could be easily identified, although in Nissl preparations the definition of these nuclei was less clear, probably owing to the presence of the status marmoratus and widespread fine and coarse glial fibrosis. The internal capsule, crura, and pyramidal tracts,

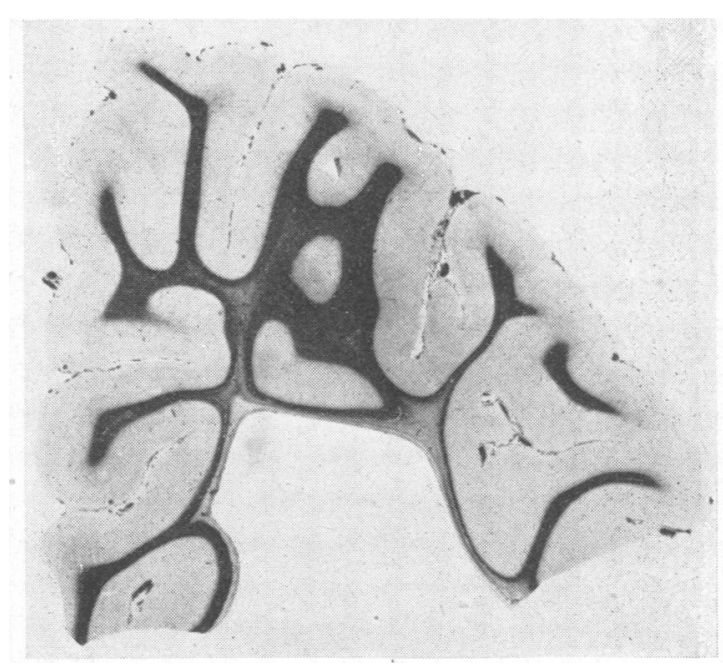

Fig. 4.-Coronal section through parietal region. Heidenhain's myelin method. $\times 1.9$.

although well myelinated, were disproportionately small, and there was considerable glial fibrosis in the capsule externa and extrema. No abnormality was seen in the cerebellum, the external granular layer of the cortex having disappeared, nor in the leptomeninges, meningeal and intracerebral blood vessels and choroid plexuses. No other anatomical anomalies were noted.

The Prenatal Cortex.-It has been possible to make a comparison between the idiot cortex and that of a premature infant.

Case 2.-This child was born after 26 weeks' gestation, weighed $678 \mathrm{~g}$., and lived for five days. The brain weighed $103 \mathrm{~g}$. Apart from a small recent haemorrhage into the body of the left caudate nucleus, the brain was not structurally abnormal. The extent of development of the cerebral sulci corresponded fairly well with that shown by Connolly (1940) in a cerebral hemisphere of this age.

It can be seen in Fig. 5 that there is a comparable horizontal lamination of cells in the outer two-thirds of the cortex, although this is not so obvious as in the idiot brain. In the latter the cells are considerably more widely spaced, the distance between the rows being increased in consequence. Also, the primitive arrangement of cells in vertical columns persists more markedly in the late foetal brain. The tendency to horizontal lamination in this brain, as in the idiot's, was more noticeable in the prefrontal region of the cortex than elsewhere.

\section{Discussion}

I was unable to find any description of a lamination of nerve cells resembling that seen in the cortex of the $2 \frac{1}{4}$ year old idiot in the literature on cortical maldevelopment or on foetal and infantile lesions. Neither is it recorded in the literature 
Fig. 5.-Frontal cortex of premature infant. Nissl. $\times 80$.

on hydrocephalus, and Professor D. S. Russell (personal communication), who was shown the slides of this case, has seen nothing resembling it in her material.

Our knowledge of the morphological aspects of the later stages of prenatal cortical development is surprisingly fragmentary. Following the work of His (1890), studies have been made more recently by Filimonoff (1929) and Fitz-Gerald (1940). None of these authors describes horizontal lamination in the cortex, nor does Conel (1939) in his study of the neonatal cortex. It is, however, apparent from photographs presented by both Filimonoff and Fitz-Gerald that irregular horizontal layers of neuroblasts may be formed at a time when they are presumably migrating through the cerebral hemisphere. In Filimonoff's photographs of the calcarine region in a $3 \frac{1}{4}$ months old human foetus such an appearance is shown in the inner dark layer intervening between the pale $Z$ wischenschicht and the densely cellular subependymal matrix, as well as in the diffuse layer of cells immediately beneath the definitive cortex. One of Fitz-Gerald's photographs also shows a horizontal lamination of cells immediately beneath the definitive cortex of a $95 \mathrm{~mm}$. human embryo.

The finding of horizontal lamination of cells in the cortex of an immature child of about 26 weeks' gestation, as in the second case described, is therefore of considerable interest as it seems to provide a clue to the interpretation of the cortical anomaly in the first case. The persistence of this arrangement in the latter, exaggerated by the subsequent increase in depth of the cortex, appears to suggest that a check had occurred to the normal process of cerebral maturation, presumably during the prenatal period. However, with regard to the occurrence - of horizontal cell lamination as a mark of immaturity, a comparative study of the cortex at birth in a large series of brains would be necessary in order to assess the degree of individual variability of maturation that may be found before evidence of this kind can be of much value in precisely dating the incidence of a damaging lesion. It should be noted that the lamination in the idiot brain is clearly confined to the upper layers of the cortex. thereby providing an example of the much disputed abnormality in the supragranular cortex in such cases (Bolton, 1914 ; Stewart, 1935 ; Norman, 1938).

From the histological study of the white matter lesion alone, no definite opinion could be expressed as to the nature of the damage which it has evidently sustained. Marked internal hydrocephalus was present, the dilation being confined to the third and lateral ventricles, although there was no expansion of the brain as a whole, or of the skull. The possibility that there had been a transient increase in the intraventricular fluid pressure produced by an obstruction either. to the circulation of cerebrospinal fluid or to the venous drainage of the choroid plexus cannot be ruled out. On the other hand the hydrocephalus may equally well have occurred ex vacuo as a consequence of the white matter shrinking after damage of an anoxic nature. The presence of what appears to be an abortive marbling in the thalamus would support an anoxic pathogenesis. Although status marmoratus of the subcortical centres is usually due to trauma sustained at birth (Norman, 1949), this does not wholly preclude its occasional onset in prenatal life (Meyer and Cook, 1936).

Whatever the pathological explanation of this lesion may be, it is probable that the cortical anomaly arose as a sequel to the white matter damage, or as part of the same process. The concentric arrangement of myelinated fibres around the ventricles invites comparison with the concentric demyelination described by Baló (1928). It was suggested by Spatz (1931) and by Hallervorden and Spatz (1933) that this marks the site of periodic precipitations accompanying the diffusion of a toxic substance through the white matter. In the present instance one might suppose that a similar diffusion had occurred from the ventricles through the ependyma into the deep cerebral white matter, which as yet had not become fully myelinated.

\section{Summary}

The brain of a spastic idiot child aged $2 \frac{1}{4}$ years is described. Anomalous horizontal lamination of nerve cells was seen in the supragranular cerebral cortex, particularly marked in the prefrontal region, associated with the presence of internal hydro- 
cephalus and widespread shrinkage and gliosis through the imperfectly myelinated white matter of both cerebral hemispheres. A comparable, although less conspicuous, laminar arrangement of cells was seen in the prefrontal cortex of the brain of a premature infant.

I am grateful to Professor Alfred Meyer for his advice and help in interpreting the histological picture in the idiot brain, to Dr. L. T. Hilliard for providing access to the Fountain Hospital case records, and to Elisabeth Beck for the photographs (except Figs. 1 and 5); also to Dr. R. M. Norman for his interest in the case, and to Dr. R. Aidin of Southmead Hospital, Bristol, for providing the premature brain.

\section{REFERENCES}

Baló, J. (1928). Arch. Neurol. Psychiat., Chicago, 19, 242.
Bolton, J. S. (1914). "The Brain in Health and Disease." London.

Conel, J. LeRoy (1939). "The Postnatal Development of the Human Cerebral Cortex". Vol. 1. Harvard University Press, Cambridge, Mass.

Connolly, C. J. (1940). Amer. J. phys. Anthrop., 26, 113.

Filimonoff, I. N. (1929). J. Psychol. Neurol., Lpz., $39,323$.

Fitz-Gerald, P. A. M. F. (1940). Contr. Embryol. Carneg. Instn., 28, 193. (No. 176).

Hallervorden, J., and Spatz, H. (1933). Arch. Psychiat. Nervenkr., 98, 641.

His, W. (1890). Arch. Anat. Physiol., Anatom. Abt., Suppl.-Bd., p. 95.

Meyer, A., and Cook, L. C. (1936). J. Neurol. Psychopath., 16, 341.

Norman, R. M. (1938). J. Neurol. Psychiat., N.S., $1,198$. (1949). Brain, 72, 83.

Spatz, H. (1931). Allg. Z. Psychiat., 96, 367.

Stewart, R. M. (1935). Proc. roy. Soc. Med., 28, 786. 